\title{
Hyperentanglement concentration for two-photon four-qubit systems with linear optics*
}

\author{
Bao-Cang Ren, Fang-Fang Du, and Fu-Guo Deng ${ }^{\dagger}$ \\ Department of Physics, Applied Optics Beijing Area Major Laboratory, \\ Beijing Normal University, Beijing 100875, China
}

(Dated: August 6, 2018)

\begin{abstract}
Hyperentanglement, defined as the entanglement in several degrees of freedom (DOFs) of a quantum system, has attracted much attention recently. Here we investigate the possibility of concentrating the two-photon four-qubit systems in partially hyperentangled states in both the spatial mode and the polarization DOFs with linear optics. We first introduce our parameter-splitting method to concentrate the systems in the partially hyperentangled states with known parameters, including partially hyperentangled Bell states and cluster states. Subsequently, we present another two nonlocal hyperentanglement concentration protocols (hyper-ECPs) for the systems in partially hyperentangled unknown states, resorting to the Schmidt projection method. It will be shown that our parameter-splitting method is very efficient for the concentration of the quantum systems in partially entangled states with known parameters, resorting to linear-optical elements only. All these four hyper-ECPs are feasible with current technology and they may be useful in long-distance quantum communication based on hyperentanglement as they require only linear optical elements.

PACS numbers: 03.67.Pp, 03.67.Bg, 03.65.Yz, 03.67.Hk
\end{abstract}

\section{INTRODUCTION}

Entanglement, a unique phenomenon in quantum mechanics theory, has largely improved the methods of manipulating and transforming information in quantum information processing and quantum computing [1]. The entangled photon systems can act as the quantum channels in some typical long-distance quantum communication proposals, such as quantum key distribution [2], quantum teleportation [3], quantum dense coding [4, 5], quantum secret sharing [6, 7], and so on. An entangled photon pair is usually produced locally, so the environment noise is inevitable for it in its distribution process among the parties in quantum communication, which will decrease its entanglement. The photon signals can only be transmitted no more than several hundreds of kilometers in an optical fiber or a free space with current technology, and quantum repeaters are required to connect the two neighboring nodes in long-distance quantum communication network. In the storage of a quantum state in a quantum repeater, the entanglement of an entangled photon system will also be decreased by decoherence. The fidelity and the security of long-distance quantum communication protocols may be decreased with the decrescence of the entanglement in photon systems.

Entanglement purification and entanglement concentration are two passive ways for depressing the noise effect on entangled systems, with which some high-fidelity nonlocal entangled systems can be obtained from a set of less-entangled systems. Entanglement purification is used to distill a subset of high-fidelity nonlocal entangled

\footnotetext{
*Published in Phys. Rev. A 88, 012302 (2013)

${ }^{\dagger}$ Corresponding author: fgdeng@bnu.edu.cn
}

systems from a set of those in a mixed state with less entanglement [8 16], while entanglement concentration is used to distill some nonlocal maximally-entangled systems from a set of systems in a partially-entangled pure state. In 1996, Bennett et al. [17] introduced the first entanglement concentration protocol (ECP) for two-photon systems by using the Schmidt projection method and collective measurements. Since this pioneering work, many interesting ECPs have been proposed for photon systems [18 27]. They can be divided into two groups. In the first group, the ECPs $[17-19,22]$ require that the two remote users, say Alice and Bob, do not know the parameters about the partially entangled pure state of the photon systems. In the second group, the ECPs [23 27] require that Alice and Bob know the parameters accurately. The latter have a higher efficiency than that in the former in theory.

Hyperentanglement, defined as the entanglement in several degrees of freedom (DOFs) of a quantum system, has attracted much attention for quantum information recently. For example, it has been used to assist the polarization photonic Bell-state analysis [28 32 ]. In 2003, Walborn et al. 32. presented a complete Bellstate analysis in the coincidence basis using hyperentangled states. In 2002, Simon and Pan [11] presented a polarization photonic entanglement purification protocol (EPP) for a parameter down-conversion (PDC) source with linear optics, resorting to spatial-polarization hyperentanglement. In 2005, Barreiro et al. [33] experimentally prepared hyperentangled photon pairs in polarization, spatial mode, and time energy DOFs with spontaneous parametric down-conversion photons. In 2008, Sheng et al. [13] presented an efficient EPP for a PDC source with nonlinear optics, resorting to the spatialpolarization hyperentanglement. Subsequently, some deterministic EPPs $[14-16]$ were proposed with hyperentan- 
glement. In 2008, Barreiro et al. [34] beat the channel capacity limit of superdense coding with linear optics, resorting to polarization-orbital-angular-momentum hyperentanglement. And some hyperentangled Bell-state analysis protocols [35 38] were proposed to increase the channel capacity of quantum communication recently. In 2007, Wei et al. [38] divided the 16 hyperentangled Bell states into seven groups with linear optics. In 2012, an interesting quantum repeater protocol based on spatialpolarization hyperentanglement was proposed [39]. In 2013, Graham et al. [40] experimentally implemented direct characterization of quantum dynamics assisted by hyperentanglement.

In this article, we investigate the possibility of concentrating the two-photon four-qubit systems in the nonlocal partially hyperentangled states in both the spatial mode and the polarization DOFs with linear-optical elements. First, we introduce two feasible nonlocal spatialpolarization hyperentanglement concentration protocols (hyper-ECPs) for the systems in partially hyperentangled states with known parameters (including hyperentangled Bell-class states and cluster-class states), resorting to our parameter-splitting method. Subsequently, we present another two hyper-ECPs for the partially hyperentangled photon systems in unknown states, resorting to the Schmidt projection method, a conventional way for entanglement concentration. These two hyper-ECPs can be accomplished with two copies of unknown states and parity-check measurements. The parity-check measurement on the polarization DOF is constructed with polarizing beam splitters and single-photon detectors, and the parity-check measurement on spatial-mode DOF is constructed with the Hong-Ou-Mandel (HOM) effect 41] of a 50:50 beam splitter and single-photon detectors. It will be shown that the hyper-ECPs with our parametersplitting method are far more efficient for the concentration of the quantum systems in partially entangled states with known parameters than those with the Schmidt projection method.

This paper is organized as follows: In Sec. III we present two hyper-ECPs for the two-photon fourqubit systems in known pure states with our parametersplitting method, including the one used for the systems in a known partially hyperentangled Bell state, which is discussed in Sec. [IA and the other used for those in a known partially hyperentangled cluster state, discussed in Sec. IIB In Sec. III we discuss the hyperentanglement concentration for the two-photon fourqubit systems in unknown partially entangled pure states with linear optics. Some discussions and a summary are given in Sec. IV] In Appendix A, a hyper-ECP for an arbitrary unknown hyperentangled cluster-class state is discussed. In Appendix $\mathrm{B}$ the entanglement purification of a mixed hyperentangled Bell state is given. Our parameter-splitting-based entanglement concentration for a known partially entangled state in one DOF is discussed in Appendix C

\section{HYPER-ECPS WITH PARAMETER-SPLITTING METHOD}

In this section, we present two hyper-ECPs for twophoton four-qubit systems in known nonlocal hyperentangled spatial-polarization pure states based on our parameter-splitting method with linear-optical elements. One hyper-ECP is used for two-photon four-qubit systems in a known partially hyperentangled Bell state and the other is used for those in a known partially hyperentangled cluster state. In both these hyper-ECPs, Alice and Bob obtain a subset of two-photon four-qubit systems in maximally hyperentangled states by splitting the parameters of the initial nonlocal partially hyperentangled states with linear-optical elements only.

\section{A. Hyper-ECP for two-photon four-qubit systems in a partially hyperentangled Bell state}

Let us assume that the initial nonlocal hyperentangled Bell-class state in both the spatial mode and the polarization DOFs is

$$
\begin{aligned}
\left|\varphi_{0}\right\rangle_{A B}= & \left(\alpha|H\rangle_{A}|H\rangle_{B}+\beta|V\rangle_{A}|V\rangle_{B}\right) \\
& \otimes\left(\gamma\left|a_{1}\right\rangle\left|b_{1}\right\rangle+\delta\left|a_{2}\right\rangle\left|b_{2}\right\rangle\right) .
\end{aligned}
$$

Here the subscripts $A$ and $B$ represent the two photons kept by the two remote users, say Alice and Bob. $|H\rangle$ and $|V\rangle$ represent the horizontal and the vertical polarizations of photons, respectively. $\left|a_{1}\right\rangle\left(\left|b_{1}\right\rangle\right)$ and $\left|a_{2}\right\rangle\left(\left|b_{2}\right\rangle\right)$ are the two spatial modes of the photon $A(B) . \alpha, \beta, \gamma$, and $\delta$ are four real parameters that are known to Alice and Bob, and they satisfy the relation

$$
|\alpha|^{2}+|\beta|^{2}=|\gamma|^{2}+|\delta|^{2}=1
$$

For describing the principle of our hyper-ECP explicitly and simply, we assume that $|\alpha|>|\beta|$ and $|\gamma|<|\delta|$ below. The principle of our hyper-ECP in other cases is the same as this one with or without a little modification.

The principle of our hyper-ECP for a partially hyperentangled Bell state is shown in Fig. 1(a). It can be implemented with some local unitary operations on the photon $A$ in both the spatial-mode and the polarization DOFs performed by Alice. Bob performs no operations in this hyper-ECP. In detail, first, Alice performs a unitary operation on the spatial mode $a_{2}$ by using an unbalanced BS (i.e., $\mathrm{UBS}_{1}$ ) [42] with the reflection coefficient $R_{1}=\gamma / \delta$, shown in Fig. 1(b), and the partially hyperentangled Bell-class state $\left|\varphi_{0}\right\rangle_{A B}$ is changed to be $\left|\varphi_{1}\right\rangle_{A B}$. Here

$$
\begin{aligned}
\left|\varphi_{1}\right\rangle_{A B}= & \left(\alpha|H\rangle_{A}|H\rangle_{B}+\beta|V\rangle_{A}|V\rangle_{B}\right) \otimes\left[\gamma \left(\left|a_{1}\right\rangle\left|b_{1}\right\rangle\right.\right. \\
& \left.\left.+\left|a_{2}\right\rangle\left|b_{2}\right\rangle\right)+\sqrt{|\delta|^{2}-|\gamma|^{2}}\left|a_{3}\right\rangle\left|b_{2}\right\rangle\right] .
\end{aligned}
$$

That is, Alice splits the parameters of the hyperentangled Bell-class state in the spatial-mode DOF with $\mathrm{UBS}_{1}$. 


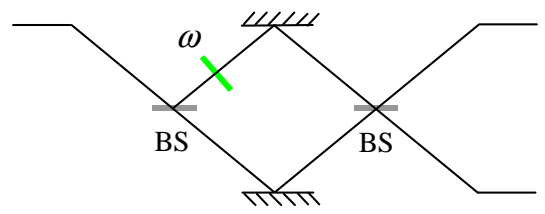

(b)
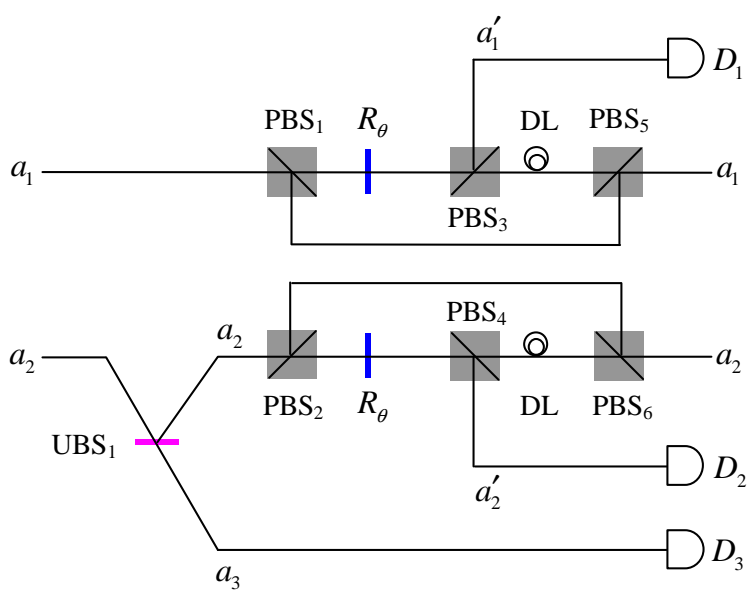

(a)

FIG. 1: (Color online) (a) Schematic diagram of our hyperECP for a partially hyperentangled Bell state with known parameters. (b) Schematic diagram of an unbalanced BS (UBS). BS represents a balanced 50:50 beam splitter. $\omega$ represents a phase shift which decides the reflection coefficient and the transmission coefficient of the unbalanced BS. $a_{1}$ and $a_{2}$ represent the two original spatial modes of the photon $A$. $a_{1}^{\prime}$ and $a_{2}^{\prime}$ are another two spatial modes of the photon $A$. UBS 1 represents an unbalanced beam splitter with the reflection coefficient $R_{1}=\gamma / \delta . R_{\theta}$ represents a wave plate which can rotate the horizontal polarization with an angle $\theta=\arccos (\beta / \alpha)$. $\mathrm{PBS}_{i}(i=1,2, \cdots, 6)$ represents a polarizing beam splitter, which transmits the photon in the horizontal polarization $|H\rangle$ and reflects the photon in the vertical polarization $|V\rangle$, respectively. DL denotes a time-delay device which is used to make the two wavepackets reach the last $\mathrm{PBS}\left(\mathrm{PBS}_{5}\right.$ or $\left.\mathrm{PBS}_{6}\right)$ in each Mach-Zehnder interferometer simultaneously. $D_{1}, D_{2}$, and $D_{3}$ represent three single-photon detectors.

One can see that the spatial-mode state of the twophoton system $A B$ becomes a maximally entangled one if the photon $A$ does not emit from the spatial mode $a_{3}$.

Second, Alice transforms the polarization-mode state of the two-photon system $A B$ into a maximally entangled one by splitting the parameters of the hyperentangled Bell-class state in the polarization DOF, if the photon $A$ does not emit from the spatial mode $a_{3}$. As shown in Fig. 1(a), Alice performs the same polarization unitary operations on the two spatial modes $a_{1}$ and $a_{2}$. The wave plate $R_{\theta}$ is used to rotate the horizontal polarization $|H\rangle$ with an angle $\theta=\arccos (\beta / \alpha)$, that is, $|H\rangle \rightarrow \cos \theta|H\rangle+$ $\sin \theta|V\rangle$. After the photon $A$ coming from the two spatial modes $a_{1}$ and $a_{2}$ passes through PBSs (i.e., $\mathrm{PBS}_{1}$ and
$\mathrm{PBS}_{2}$ ) and $R_{\theta}$, the state of the system is transformed from $\left|\varphi_{1}\right\rangle_{A B}$ into $\left|\varphi_{2}\right\rangle_{A B}$. Here

$$
\begin{aligned}
\left|\varphi_{2}\right\rangle_{A B}= & {\left[\beta\left(|H\rangle_{A}|H\rangle_{B}+|V\rangle_{A}|V\rangle_{B}\right)\right.} \\
& \left.+\sqrt{|\alpha|^{2}-|\beta|^{2}}\left|V^{\prime}\right\rangle_{A}|H\rangle_{B}\right] \\
& \otimes \gamma\left(\left|a_{1}\right\rangle\left|b_{1}\right\rangle+\left|a_{2}\right\rangle\left|b_{2}\right\rangle\right) \\
& +\left(\alpha|H\rangle_{A}|H\rangle_{B}+\beta|V\rangle_{A}|V\rangle_{B}\right) \\
& \otimes \sqrt{|\delta|^{2}-|\gamma|^{2}}\left|a_{3}\right\rangle\left|b_{2}\right\rangle .
\end{aligned}
$$

Here $\left|V^{\prime}\right\rangle$ presents the vertical polarization of the photon after the operation $R_{\theta}$.

Third, when the photon A passes through $\mathrm{PBS}_{3}$ $\left(\mathrm{PBS}_{4}\right), \mathrm{DL}$, and $\mathrm{PBS}_{5}\left(\mathrm{PBS}_{6}\right)$, the state of the twophoton system is transformed from $\left|\varphi_{2}\right\rangle_{A B}$ into $\left|\varphi_{3}\right\rangle_{A B}$. Here

$$
\begin{aligned}
\left|\varphi_{3}\right\rangle_{A B}= & \beta \gamma(|H\rangle|H\rangle+|V\rangle|V\rangle)_{A B}\left(\left|a_{1}\right\rangle\left|b_{1}\right\rangle+\left|a_{2}\right\rangle\left|b_{2}\right\rangle\right) \\
& +\gamma \sqrt{|\alpha|^{2}-|\beta|^{2}}|V\rangle_{A}|H\rangle_{B}\left(\left|a_{1}^{\prime}\right\rangle\left|b_{1}\right\rangle+\left|a_{2}^{\prime}\right\rangle\left|b_{2}\right\rangle\right) \\
& +\sqrt{|\delta|^{2}-|\gamma|^{2}}(\alpha|H\rangle|H\rangle+\beta|V\rangle|V\rangle)_{A B}\left|a_{3}\right\rangle\left|b_{2}\right\rangle .
\end{aligned}
$$

From Eq. (5), one can see that the state of the twophoton system $A B$ becomes a maximally hyperentangled Bell state $\left|\varphi_{f}\right\rangle_{A B}$ if the photon $A$ emits from the spatial modes $a_{1}$ and $a_{2}$. Here

$$
\left|\varphi_{f}\right\rangle_{A B}=\frac{1}{2}(|H\rangle|H\rangle+|V\rangle|V\rangle)_{A B}\left(\left|a_{1}\right\rangle\left|b_{1}\right\rangle+\left|a_{2}\right\rangle\left|b_{2}\right\rangle\right) .(6)
$$

If the photon $A$ emits from the spatial mode $a_{1}^{\prime}, a_{2}^{\prime}$, or $a_{3}$, Alice and Bob cannot obtain a maximally hyperentangled Bell state $\left|\varphi_{f}\right\rangle_{A B}$, which means this hyper-ECP fails. In theory, Alice can judge whether this hyper-ECP succeeds or not, according to the spatial mode of the photon $A$.

It is not difficult to calculate the success probability of our hyper-ECP for a hyperentangled Bell-class state. If the photon $A$ does not emit from the spatial mode $a_{1}^{\prime}$, $a_{2}^{\prime}$, and $a_{3}$, our hyper-ECP succeeds, which takes place with the probability of $P_{1}=4|\beta \gamma|^{2}$. Otherwise, the photon $A$ is detected by the single-photon detectors and is destroyed, and our hyper-ECP fails. That is, the total success probability of our hyper-ECP is $P_{1}=4|\beta \gamma|^{2}$, shown in Fig. 2 ,

In a practical application of our hyper-ECP, there are two ways for Alice and Bob, the two parties in quantum communication to judge whether this hyper-ECP succeeds or not. On one hand, Alice can judge that this hyper-ECP fails if one of the three detectors $D_{1}, D_{2}$, and $D_{3}$ is clicked by the photon $A$. On the other hand, if the efficiency of the single-photon detectors $D_{i}(i=1,2,3)$ is $100 \%$, Alice can also judge that this hyper-ECP succeeds if there are no single-photon detectors clicked. At present, the efficiency of a single-photon detector is lower than $100 \%$. That is, the case that there is a photon which should be detected by the single-photon detectors but not detected because of detection inefficiency, can be mistaken as a successful event if Alice judges whether this 
hyper-ECP succeeds or not with only the single-photon detectors shown in Fig. 11. Fortunately, this mistaken case can be eliminated by postselection, as the same as the entanglement purification protocols $10-12,14,16$ ] and the entanglement concentration protocols [18 21] in only one DOF with linear-optical elements. That is, this hyper-ECP succeeds if Alice detects the photon $A$ emitting from either the spatial modes $a_{1}$ or $a_{2}$, when Alice and Bob use the photon pair $A B$ to complete their task in quantum communication. Although the photon pair $A B$ are detected in this time, the task of quantum communication is also accomplished.

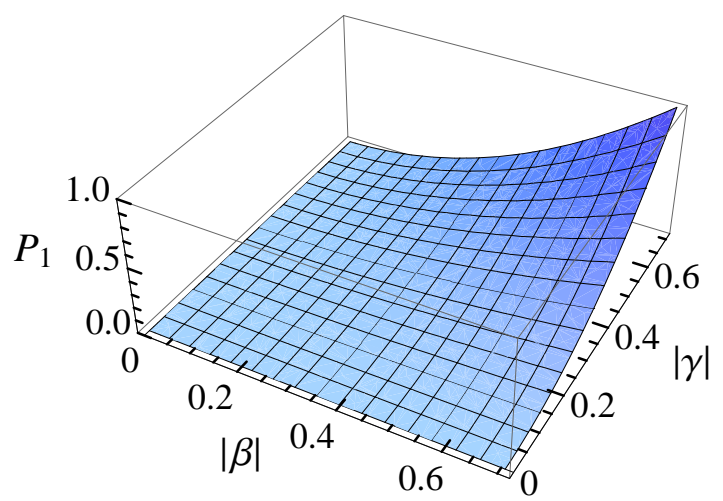

FIG. 2: (Color online) The success probability of our hyperECP for each two-photon four-qubit system in a partially hyperentangled Bell-class state with known parameters.

\section{B. Hyper-ECP for two-photon four-qubit systems in a partially hyperentangled cluster state}

Suppose that the initial two-photon four-qubit partially hyperentangled cluster-class state shared by two remote users Alice and Bob is

$$
\begin{aligned}
\left|\psi_{0}\right\rangle_{A B}= & \alpha|H\rangle_{A}|H\rangle_{B}\left|a_{1}\right\rangle\left|b_{1}\right\rangle+\beta|V\rangle_{A}|V\rangle_{B}\left|a_{1}\right\rangle\left|b_{1}\right\rangle \\
& +\gamma|H\rangle_{A}|H\rangle_{B}\left|a_{2}\right\rangle\left|b_{2}\right\rangle-\delta|V\rangle_{A}|V\rangle_{B}\left|a_{2}\right\rangle\left|b_{2}\right\rangle
\end{aligned}
$$

The four parameters $\alpha, \beta, \gamma$, and $\delta$ are known to Alice and Bob, and they satisfy the relation

$$
|\alpha|^{2}+|\beta|^{2}+|\gamma|^{2}+|\delta|^{2}=1
$$

We assume $|\gamma|>|\alpha|>|\beta|>|\delta|$ below for describing the principle of our hyper-ECP explicitly.

The principle of our hyper-ECP for a partially hyperentangled two-photon four-qubit cluster-class state is shown in Fig. 3, which is also implemented with linearoptical elements only, by splitting the parameters of the cluster-class state $\left|\psi_{0}\right\rangle_{A B}$. This hyper-ECP can also be accomplished with local unitary operations on the photon $A$ in both the spatial-mode and the polarization DOFs, and it includes three steps, as shown in Fig. 3 .
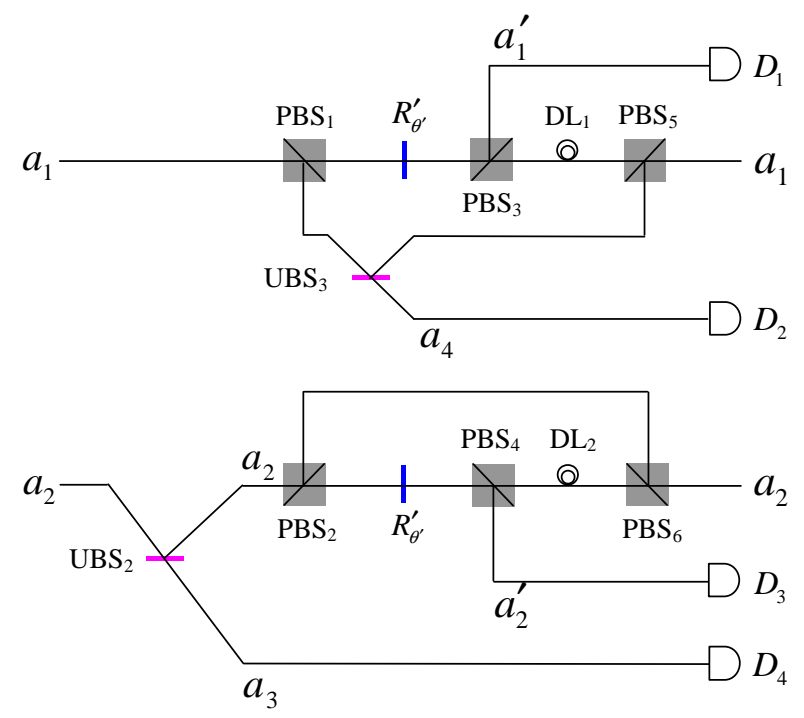

FIG. 3: (Color online) Schematic diagram of our hyperECP for partially-entangled two-photon four-qubit clusterclass states with known parameters. $\mathrm{UBS}_{2}$ and $\mathrm{UBS}_{3}$ represent two unbalanced beam splitters with the reflection coefficients $R_{2}=\alpha / \gamma$ and $R_{3}=(\alpha \delta) /(\gamma \beta)$, respectively. $R_{\theta^{\prime}}^{\prime}$ represents a wave plate which can rotate the horizontal polarization with an angle $\theta^{\prime}=\arccos (\delta / \gamma)$. $\mathrm{DL}_{1}\left(\mathrm{DL}_{2}\right)$ denotes a time-delay device which is used to make the two wavepackets reach the last $\mathrm{PBS}\left(\mathrm{PBS}_{5}\right.$ or $\left.\mathrm{PBS}_{6}\right)$ in each Mach-Zehnder interferometer simultaneously.

In the first step, only a spatial-mode unitary operation is performed on the photon $A$ from the spatial mode $a_{2}$ by using an unbalanced BS $\left(\mathrm{UBS}_{2}\right)$ with the reflection coefficient $R_{2}=\alpha / \gamma$. The partially-entangled twophoton four-qubit cluster state $\left|\psi_{0}\right\rangle_{A B}$ is transformed into $\left|\psi_{1}\right\rangle_{A B}$ in this step. Here

$$
\begin{aligned}
\left|\psi_{0}\right\rangle_{A B}= & \alpha\left|a_{1}\right\rangle\left|b_{1}\right\rangle\left(|H\rangle_{A}|H\rangle_{B}+\frac{\beta}{\alpha}|V\rangle_{A}|V\rangle_{B}\right) \\
& +\gamma\left|a_{2}\right\rangle\left|b_{2}\right\rangle\left(|H\rangle_{A}|H\rangle_{B}-\frac{\delta}{\gamma}|V\rangle_{A}|V\rangle_{B}\right) \\
\left|\psi_{1}\right\rangle_{A B}= & \alpha\left[\left|a_{1}\right\rangle\left|b_{1}\right\rangle\left(|H\rangle_{A}|H\rangle_{B}+\frac{\beta}{\alpha}|V\rangle_{A}|V\rangle_{B}\right)\right. \\
& \left.+\left|a_{2}\right\rangle\left|b_{2}\right\rangle\left(|H\rangle_{A}|H\rangle_{B}-\frac{\delta}{\gamma}|V\rangle_{A}|V\rangle_{B}\right)\right] \\
& +\sqrt{|\gamma|^{2}-|\alpha|^{2}}\left|a_{3}\right\rangle\left|b_{2}\right\rangle\left(|H\rangle_{A}|H\rangle_{B}\right. \\
& \left.-\frac{\delta}{\gamma}|V\rangle_{A}|V\rangle_{B}\right)
\end{aligned}
$$

In the second step, Alice first performs a unitary operation with $\mathrm{PBS}_{1}$ on the photon $A$ emitting from the spatial mode $a_{1}$ and then Alice performs a unitary operation on the photon $A$ coming from the vertical polarization path of the spatial mode $a_{1}$, by using an unbalanced BS $\left(\mathrm{UBS}_{3}\right)$ with the reflection coefficient $R_{3}=(\alpha \delta) /(\gamma \beta)$. These two operations transform the partially-entangled 
state $\left|\psi_{1}\right\rangle_{A B}$ into $\left|\psi_{2}\right\rangle_{A B}$. Here,

$$
\begin{aligned}
\left|\psi_{2}\right\rangle_{A B}= & \alpha\left[|H\rangle_{A}|H\rangle_{B}\left(\left|a_{1}\right\rangle\left|b_{1}\right\rangle+\left|a_{2}\right\rangle\left|b_{2}\right\rangle\right)\right. \\
& +\frac{\delta}{\gamma}|V\rangle_{A}|V\rangle_{B}\left(\left|a_{1}\right\rangle\left|b_{1}\right\rangle-\left|a_{2}\right\rangle\left|b_{2}\right\rangle\right] \\
& +\alpha \sqrt{\left|\frac{\beta}{\alpha}\right|^{2}-\left|\frac{\delta}{\gamma}\right|^{2}}|V\rangle_{A}|V\rangle_{B}\left|a_{4}\right\rangle\left|b_{1}\right\rangle \\
& +\sqrt{|\gamma|^{2}-|\alpha|^{2}}\left|a_{3}\right\rangle\left|b_{2}\right\rangle\left(|H\rangle_{A}|H\rangle_{B}\right. \\
& \left.-\frac{\delta}{\gamma}|V\rangle_{A}|V\rangle_{B}\right) .
\end{aligned}
$$

The last step is used to perform unitary operations on the photon $A$ in the polarization modes. As shown in Fig. 3. the polarization unitary operations performed on the photon $A$ by Alice are the same ones for the two spatial modes $a_{1}$ and $a_{2}$, in which $R_{\theta^{\prime}}^{\prime}$ contributes a rotation angle $\theta^{\prime}=\arccos (\delta / \gamma)$ on the horizontal polarization of the photon $A$. After photon $A$ passes through $R_{\theta^{\prime}}^{\prime}$ and PBS, the state $\left|\psi_{2}\right\rangle_{A B}$ is changed to be $\left|\psi_{3}\right\rangle_{A B}$. Here

$$
\begin{aligned}
\left|\psi_{3}\right\rangle_{A B}= & \frac{\alpha \delta}{\gamma}\left[|H\rangle_{A}|H\rangle_{B}\left(\left|a_{1}\right\rangle\left|b_{1}\right\rangle+\left|a_{2}\right\rangle\left|b_{2}\right\rangle\right)\right. \\
& +|V\rangle_{A}|V\rangle_{B}\left(\left|a_{1}\right\rangle\left|b_{1}\right\rangle-\left|a_{2}\right\rangle\left|b_{2}\right\rangle\right] \\
& +\alpha \sqrt{1-\left|\frac{\delta}{\gamma}\right|^{2}}|V\rangle_{A}|H\rangle_{B}\left(\left|a_{1}^{\prime}\right\rangle\left|b_{1}\right\rangle+\left|a_{2}^{\prime}\right\rangle\left|b_{2}\right\rangle\right) \\
& +\alpha \sqrt{\left|\frac{\beta}{\alpha}\right|^{2}-\left|\frac{\delta}{\gamma}\right|^{2}}|V\rangle_{A}|V\rangle_{B}\left|a_{4}\right\rangle\left|b_{1}\right\rangle \\
& +\sqrt{|\gamma|^{2}-|\alpha|^{2}}\left|a_{3}\right\rangle\left|b_{2}\right\rangle\left(|H\rangle_{A}|H\rangle_{B}\right. \\
& \left.-\frac{\delta}{\gamma}|V\rangle_{A}|V\rangle_{B}\right) .
\end{aligned}
$$

From Eq. (11), one can see that the state of the twophoton system $A B$ becomes a maximally hyperentangled cluster state $\left|\psi_{f}\right\rangle_{A B}$ if the photon $A$ emits from the spatial modes $a_{1}$ and $a_{2}$. Here

$$
\begin{aligned}
\left|\psi_{f}\right\rangle_{A B}= & \frac{1}{2}\left[|H\rangle_{A}|H\rangle_{B}\left(\left|a_{1}\right\rangle\left|b_{1}\right\rangle+\left|a_{2}\right\rangle\left|b_{2}\right\rangle\right)\right. \\
& \left.+|V\rangle_{A}|V\rangle_{B}\left(\left|a_{1}\right\rangle\left|b_{1}\right\rangle-\left|a_{2}\right\rangle\left|b_{2}\right\rangle\right)\right] .
\end{aligned}
$$

If the photon $A$ emits from the spatial mode $a_{1}^{\prime}, a_{2}^{\prime}, a_{3}$, or $a_{4}$, Alice and Bob cannot obtain a maximally hyperentangled cluster state $\left|\psi_{f}\right\rangle_{A B}$, which means this hyperECP fails. In theory, Alice can judge whether this hyperECP succeeds or not, according to the spatial modes of the photon $A$. As the same as that in our hyper-ECP for a partially hyperentangled Bell state, Alice and Bob can also judge whether this hyper-ECP succeeds or fails by postselection if the efficiencies of the single-photon detectors are low than $100 \%$ in a practical application.

It is not difficult to calculate the success probability of our hyper-ECP for a hyper-entangled two-photon clusterclass state. If one of the four detectors $D_{1}, D_{2}, D_{3}$, and $D_{4}$ clicks, at least one DOF of the partially entangled state is projected into a product state, which means that this hyper-ECP fails. If none of the four detectors $D_{1}, D_{2}, D_{3}$, and $D_{4}$ clicks in principle, the partially entangled cluster-class state $\left|\psi_{0}\right\rangle_{A B}$ is transformed into the maximally entangled two-photon four-qubit cluster state $\left|\psi_{f}\right\rangle_{A B}$ with a success probability of $P_{2}=4|\alpha \delta / \gamma|^{2}$, shown in Fig. 4 .

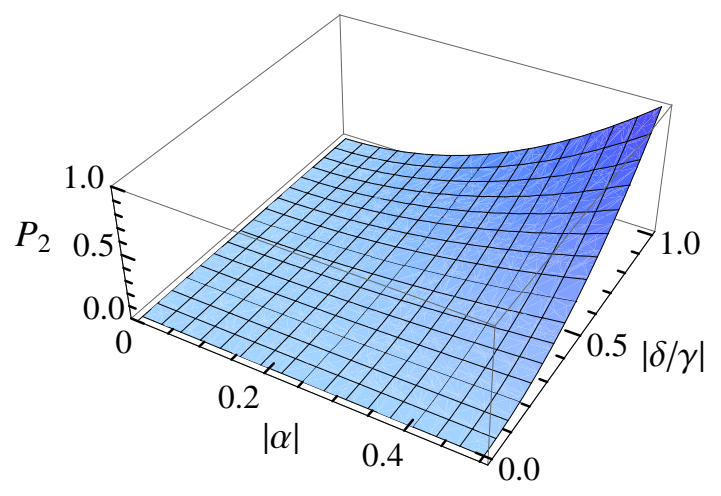

FIG. 4: (Color online) The success probability of our hyperECP for each two-photon four-qubit system in a partially hyperentangled cluster-class state with known parameters.

\section{HYPER-ECP FOR TWO-PHOTON FOUR-QUBIT SYSTEMS IN AN UNKNOWN HYPERENTANGLED PURE STATE}

In this section, we will discuss the hyper-ECPs for partially-entangled two-photon four-qubit pure states in both the spatial-mode and the polarization DOFs with their parameters unknown to the two remote users Alice and Bob, including partially hyperentangled Bell states and cluster states. In this time, the parameter-splitting method does not work as the parameters are unknown to Alice and Bob. However, these hyper-ECPs can be accomplished with the Schmidt projection method, although they are more complex than those [17 19] for the photon systems in only partially entangled polarization states.

\section{A. Hyper-ECP for two-photon four-qubit systems in an unknown hyperentangled Bell-class state}

Suppose that there are two identical two-photon systems $A B$ and $C D$ in a partially hyperentangled Bell-class state

$$
\begin{aligned}
\left|\phi_{0}\right\rangle_{A B}= & \left(\alpha|H\rangle_{A}|H\rangle_{B}+\beta|V\rangle_{A}|V\rangle_{B}\right) \\
& \otimes\left(\gamma\left|a_{1}\right\rangle\left|b_{1}\right\rangle+\delta\left|a_{2}\right\rangle\left|b_{2}\right\rangle\right), \\
\left|\phi_{0}\right\rangle_{C D}= & \left(\alpha|H\rangle_{C}|H\rangle_{D}+\beta|V\rangle_{C}|V\rangle_{D}\right) \\
& \otimes\left(\gamma\left|c_{1}\right\rangle\left|d_{1}\right\rangle+\delta\left|c_{2}\right\rangle\left|d_{2}\right\rangle\right) .
\end{aligned}
$$

Here subscripts $A$ and $B$ represent the two photons in a partially hyperentangled state $\left|\phi_{0}\right\rangle_{A B}$, and the subscripts 

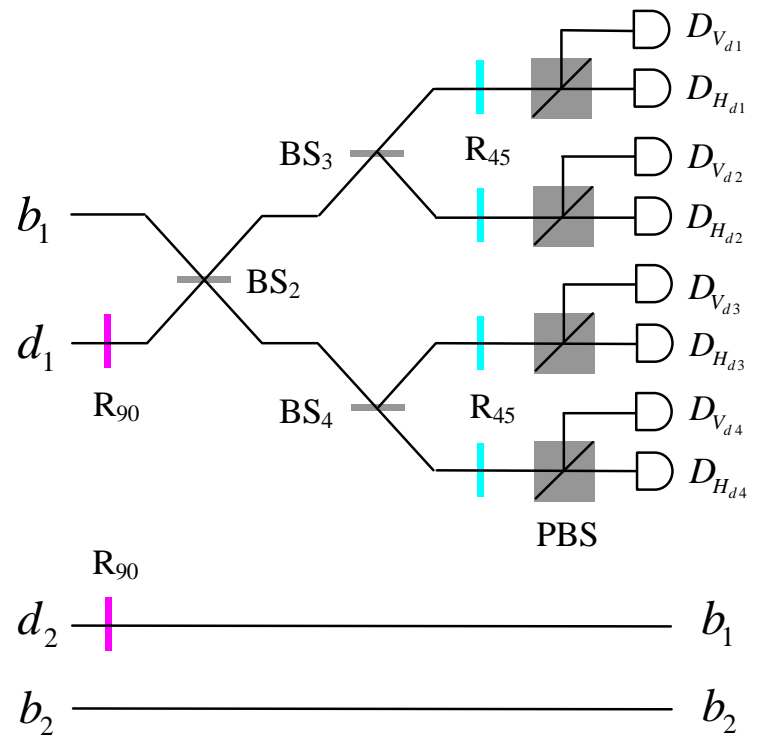

(b)

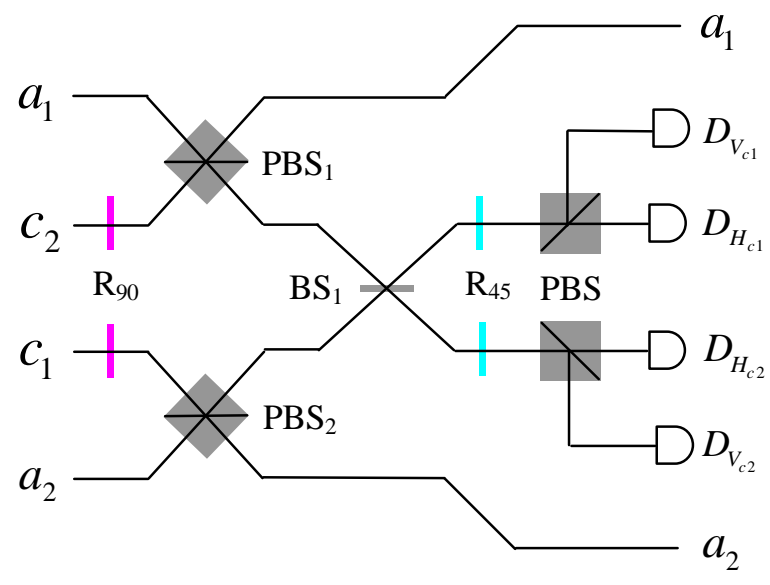

(a)

FIG. 5: (Color online) Schematic diagram of our hyper-ECP for partially hyperentangled Bell states with unknown parameters. (a) Operations performed by Alice. (b) Operations performed by Bob. $R_{90}$ represents a half-wave plate which is used to perform a polarization bit-flip operation $X=|H\rangle\langle V|+| V\rangle\langle H| . \mathrm{R}_{45}$ represents a half-wave plate which is used to perform a Hadamard operation on the polarization DOF of photons.

$C$ and $D$ represent another two photons in the partially hyperentangled state $\left|\phi_{0}\right\rangle_{C D} . i_{1}$ and $i_{2}$ are the two spatial modes of the photon $i(i=A, B, C, D)$. The photons $A$ and $C$ belong to Alice, and the photons $B$ and $D$ belong to Bob. The four parameters $\alpha, \beta, \gamma$, and $\delta$ are unknown to Alice and Bob, and they satisfy the relation $|\alpha|^{2}+|\beta|^{2}=|\gamma|^{2}+|\delta|^{2}=1$.

The principle of our hyper-ECP for partially hyper- entangled Bell-class states with unknown parameters is shown in Fig. 5. The initial state of the four-photon system $A B C D$ can be rewritten as follows:

$$
\begin{aligned}
\left|\Phi_{0}\right\rangle= & \left|\phi_{0}\right\rangle_{A B} \otimes\left|\phi_{0}\right\rangle_{C D} \\
= & \left(\alpha^{2}|H\rangle_{A}|H\rangle_{B}|H\rangle_{C}|H\rangle_{D}\right. \\
& +\alpha \beta|V\rangle_{A}|V\rangle_{B}|H\rangle_{C}|H\rangle_{D} \\
& +\alpha \beta|H\rangle_{A}|H\rangle_{B}|V\rangle_{C}|V\rangle_{D} \\
& \left.+\beta^{2}|V\rangle_{A}|V\rangle_{B}|V\rangle_{C}|V\rangle_{D}\right) \\
& \otimes\left(\gamma^{2}\left|a_{1}\right\rangle\left|b_{1}\right\rangle\left|c_{1}\right\rangle\left|d_{1}\right\rangle+\gamma \delta\left|a_{2}\right\rangle\left|b_{2}\right\rangle\left|c_{1}\right\rangle\left|d_{1}\right\rangle\right. \\
& \left.+\gamma \delta\left|a_{1}\right\rangle\left|b_{1}\right\rangle\left|c_{2}\right\rangle\left|d_{2}\right\rangle+\delta^{2}\left|a_{2}\right\rangle\left|b_{2}\right\rangle\left|c_{2}\right\rangle\left|d_{2}\right\rangle\right) .
\end{aligned}
$$

After Alice and Bob flip the polarizations of the photons $C$ and $D$ on both the two spatial modes with half wave plates $\left(R_{90}\right)$, the state of the four-photon system becomes

$$
\begin{aligned}
\left|\Phi_{1}\right\rangle= & \left(\alpha^{2}|H\rangle_{A}|H\rangle_{B}|V\rangle_{C}|V\rangle_{D}\right. \\
& +\alpha \beta|V\rangle_{A}|V\rangle_{B}|V\rangle_{C}|V\rangle_{D} \\
& +\alpha \beta|H\rangle_{A}|H\rangle_{B}|H\rangle_{C}|H\rangle_{D} \\
& \left.+\beta^{2}|V\rangle_{A}|V\rangle_{B}|H\rangle_{C}|H\rangle_{D}\right) \\
& \otimes\left(\gamma^{2}\left|a_{1}\right\rangle\left|b_{1}\right\rangle\left|c_{1}\right\rangle\left|d_{1}\right\rangle+\gamma \delta\left|a_{2}\right\rangle\left|b_{2}\right\rangle\left|c_{1}\right\rangle\left|d_{1}\right\rangle\right. \\
& \left.+\gamma \delta\left|a_{1}\right\rangle\left|b_{1}\right\rangle\left|c_{2}\right\rangle\left|d_{2}\right\rangle+\delta^{2}\left|a_{2}\right\rangle\left|b_{2}\right\rangle\left|c_{2}\right\rangle\left|d_{2}\right\rangle\right) .
\end{aligned}
$$

Subsequently, Alice puts the photons from the spatial modes $a_{1}$ and $c_{2}$ into $\mathrm{PBS}_{1}$, and those emitting from $a_{2}$ and $c_{1}$ into $\mathrm{PBS}_{2}$. Bob puts the photons emitting from the spatial modes $b_{1}$ and $d_{1}$ into $\mathrm{BS}_{2}$. Here PBSs in Fig. 5(a) are used to complete a parity-check measurement on the polarization DOF of the two photons, and BS in Fig. 5(b) is used to complete a parity-check measurement on the spatial-mode DOF of the two photons with the HOM effect. In detail, if the two photons $A$ and $C$ have the same polarizations $|H\rangle_{A}|H\rangle_{C}$ or $|V\rangle_{A}|V\rangle_{C}$ (called each of them an even-parity state in the polarization DOF), there is one and only one photon which will be detected by the single-photon detectors shown in Fig. 5(a) in principle after the two photons pass through the PBSs $\left(\mathrm{PBS}_{1}\right.$ and $\mathrm{PBS}_{2}$ ). Otherwise, there are none or two photons which will be detected by the detectors in principle if the two photons have different polarization states (called them the odd-parity polarization states). If the two photons $B$ and $D$ have different spatial-mode states (the odd-parity states), there is one and only one photon which will be detected by the single-photon detectors shown in Fig. 5(b) in principle.

These parity-check measurements by PBSs and BSs divide the states of the four-photon system into two groups, based on both the spatial mode and the polarization DOFs. Alice and Bob pick up the even-parity terms of the polarization DOF of the photon pair $A C$ in Alice's hand with the same parameter and the odd-parity terms of the spatial-mode DOF of the photon pair $C D$ in Bob's hand with the same parameter (these instances will lead the fact that both Alice and Bob will have only one detector clicked). That is, the state of the four-photon system with the selected terms becomes

$$
\left|\Phi_{2}\right\rangle=\frac{1}{2}\left(|V\rangle_{A}|V\rangle_{B}|V\rangle_{C}|V\rangle_{D}+|H\rangle_{A}|H\rangle_{B}|H\rangle_{C}|H\rangle_{D}\right)
$$




$$
\otimes\left(\left|a_{2}\right\rangle\left|b_{2}\right\rangle\left|c_{1}\right\rangle\left|d_{1}\right\rangle+\left|a_{1}\right\rangle\left|b_{1}\right\rangle\left|c_{2}\right\rangle\left|d_{2}\right\rangle\right)
$$

In the other cases, this hyper-ECP fails.

At last, both Alice and Bob perform Hadamard operations on the spatial-mode and the polarization DOFs of the photons $C$ and $D$ with a half-wave plate $R_{45}$ and a BS, respectively. The selected terms shown in Eq. (16) are transformed into

$$
\begin{aligned}
\left|\Phi_{3}\right\rangle= & \frac{1}{4}\left[(|V\rangle|V\rangle+|H\rangle|H\rangle)_{A B}(|V\rangle|V\rangle+|H\rangle|H\rangle)_{C D}\right. \\
& \left.+(|H\rangle|H\rangle-|V\rangle|V\rangle)_{A B}(|H\rangle|V\rangle+|V\rangle|H\rangle)_{C D}\right] \\
& \otimes\left[\left(\left|a_{2}\right\rangle\left|b_{2}\right\rangle+\left|a_{1}\right\rangle\left|b_{1}\right\rangle\right)\left(\left|c_{1}\right\rangle\left|d_{1}\right\rangle+\left|c_{2}\right\rangle\left|d_{2}\right\rangle\right)\right. \\
& \left.-\left(\left|a_{1}\right\rangle\left|b_{1}\right\rangle-\left|a_{2}\right\rangle\left|b_{2}\right\rangle\right)\left(\left|c_{1}\right\rangle\left|d_{2}\right\rangle+\left|c_{2}\right\rangle\left|d_{1}\right\rangle\right)\right] .
\end{aligned}
$$

If the outcomes of the two clicked detectors are in the even-parity polarization modes and the even-parity spatial modes, the state of photon pair $A B$ is projected into the maximally Bell hyperentangled state $\left|\varphi_{f}\right\rangle_{A B}=$ $\frac{1}{2}\left(|H\rangle_{A}|H\rangle_{B}+|V\rangle_{A}|V\rangle_{B}\right)\left(\left|a_{1}\right\rangle\left|b_{1}\right\rangle+\left|a_{2}\right\rangle\left|b_{2}\right\rangle\right)$. If it is an odd-parity outcome in the measurement on the photon pair $C D$ in the polarization (spatial-mode) DOF, a phase-flip operation $\sigma_{z}^{p}=|H\rangle\langle H|-| V\rangle\langle V|\left(\sigma_{z}^{s}=\right.$ $\left.\left|b_{1}\right\rangle\left\langle b_{1}|-| b_{2}\right\rangle\left\langle b_{2}\right|\right)$ on the photon $B$ is required to obtain the state $\left|\varphi_{f}\right\rangle_{A B}$.

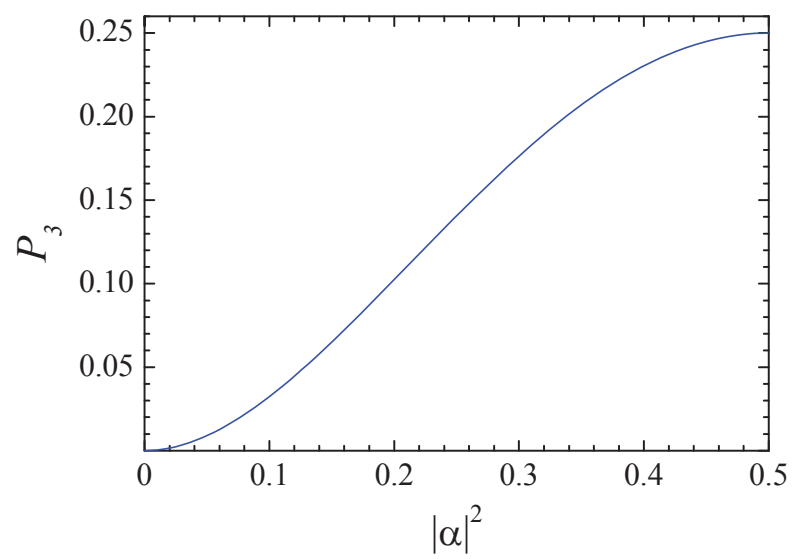

FIG. 6: (Color online) Success probability of our hyper-ECP for a pair of two-photon systems in a partially hyperentangled Bell state with unknown parameters. The relations of the parameters of the partially hyperentangled Bell state are chosen as $|\alpha|=|\gamma|$ and $|\beta|=|\delta|$.

In principle, if both Alice and Bob have only one detector clicked, the hyper-ECP succeeds with the probability $P_{3}=4|\alpha \beta \gamma \delta|^{2}$ (shown in Fig. 6 for the cases with $|\alpha|=|\gamma|$ and $|\beta|=|\delta|)$. Otherwise, the hyperECP fails. In a practical application of this hyper-ECP, Alice and Bob can also judge whether this hyper-ECP succeeds or fails by postselection if the efficiencies of the single-photon detectors are not $100 \%$.

\section{B. Hyper-ECP for two-photon four-qubit systems in an unknown hyperentangled cluster-class state}

We assume that there are two identical two-photon four-qubit systems in the partially Bell-type hyperentangled cluster-class states,

$$
\begin{aligned}
\left|\Psi_{0}\right\rangle_{A B}= & \alpha|H\rangle_{A}|H\rangle_{B}\left(\gamma\left|a_{1}\right\rangle\left|b_{1}\right\rangle+\delta\left|a_{2}\right\rangle\left|b_{2}\right\rangle\right) \\
& +\beta|V\rangle_{A}|V\rangle_{B}\left(\gamma\left|a_{1}\right\rangle\left|b_{1}\right\rangle-\delta\left|a_{2}\right\rangle\left|b_{2}\right\rangle\right) \\
\left|\Psi_{0}\right\rangle_{C D}= & \alpha|H\rangle_{C}|H\rangle_{D}\left(\gamma\left|c_{1}\right\rangle\left|d_{1}\right\rangle+\delta\left|c_{2}\right\rangle\left|d_{2}\right\rangle\right) \\
& +\beta|V\rangle_{C}|V\rangle_{D}\left(\gamma\left|c_{1}\right\rangle\left|d_{1}\right\rangle-\delta\left|c_{2}\right\rangle\left|d_{2}\right\rangle\right)
\end{aligned}
$$

Here the subscripts $A, B, C$, and $D$ represent four photons. The two photons $A$ and $C$ belong to Alice, and the two photons $B$ and $D$ belong to Bob. The four parameters $\alpha, \beta, \gamma$, and $\delta$ are unknown to Alice and Bob, and they satisfy the relation,

$$
|\alpha|^{2}+|\beta|^{2}=|\gamma|^{2}+|\delta|^{2}=1
$$

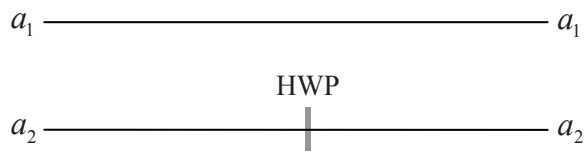

FIG. 7: Schematic diagram of the operation for transforming a maximally hyperentangled Bell state into a maximally hyperentangled two-photon cluster state. HWP represents a half-wave plate which is used to perform a polarization phaseflip operation $U_{p}=|H\rangle\langle H|-| V\rangle\langle V|$ on the photon when it emits from the spatial mode $a_{2}$.

It is suitable to implement this hyper-ECP for an unknown partially hyperentangled two-photon four-qubit cluster-class state with the quantum circuit shown in Fig. 5. Alice and Bob perform the polarization paritycheck measurement and the spatial-mode parity-check measurement on the two photon pairs $A C$ and $B D$, respectively. The even-parity terms of the polarization DOF of the photon pair in Alice's hand and the oddparity terms of the spatial-mode DOF of the photon pair in Bob's hand are picked up. After the detections and the local phase-flip operations, the state of the two-photon system $A B$ becomes $\left|\varphi_{f}\right\rangle=\frac{1}{2}\left(|H\rangle_{A}|H\rangle_{B}+\right.$ $\left.|V\rangle_{A}|V\rangle_{B}\right) \otimes\left(\left|a_{1}\right\rangle\left|b_{1}\right\rangle+\left|a_{2}\right\rangle\left|b_{2}\right\rangle\right)$ which is a maximally hyperentangled Bell state. This maximally hyperentangled Bell state can be transformed into a nonlocal maximally entangled two-photon four-qubit cluster state $\left|\psi_{f}\right\rangle_{A B}=\frac{1}{2}\left(|H\rangle_{A}|H\rangle_{B}\left|a_{1}\right\rangle\left|b_{1}\right\rangle+|V\rangle_{A}|V\rangle_{B}\left|a_{1}\right\rangle\left|b_{1}\right\rangle+\right.$ $\left.|H\rangle_{A}|H\rangle_{B}\left|a_{2}\right\rangle\left|b_{2}\right\rangle-|V\rangle_{A}|V\rangle_{B}\left|a_{2}\right\rangle\left|b_{2}\right\rangle\right)$ with the operation shown in Fig. 7 .

Up to now, we have discussed the hyper-ECP for a partially hyperentangled Bell-type two-photon four-qubit cluster-class state and its success probability is $P_{4}=$ $4|\alpha \beta \gamma \delta|^{2}$ with only linear-optical elements and singlephoton detectors, the same as that in the hyper-ECP 
for a hyperentangled Bell-class state with unknown parameters. The hyperentanglement concentration of twophoton four-qubit systems in an arbitrary unknown partially hyperentangled cluster-class state is more complex than that in a partially hyperentangled Bell-type twophoton four-qubit cluster-class state, as discussed in Appendix A. In a practical application, Alice and Bob can first transmit a hyperentangled Bell state over a quantum channel and concentrate it with the hyper-ECP discussed in Sec. IIIA and then they transform a nonlocal maximally hyperentangled Bell state into a nonlocal maximally hyperentangled two-photon four-qubit cluster state. This modification can reduce the resource needed.

\section{DISCUSSION AND SUMMARY}

We have proposed four hyper-ECPs for two classes of two-photon four-qubit states partially entangled in both the spatial-mode and the polarization DOFs of twophoton systems in two kinds of conditions with linearoptical elements. The two classes of two-photon states are hyperentangled Bell states and cluster states, respectively, and the two kinds of conditions discussed are the two-photon states with known parameters and those with unknown parameters, respectively.

In the first kind of condition about the two-photon four-qubit states with their parameters known to the two remote legitimate users, only one of the two parties is required to perform local operations in our hyper-ECPs. Here the unbalanced BS and the half wave plates for rotating the horizontal polarization are required to decrease the parameters of the terms with large probabilities until they are equal to the parameters of the terms with the smallest probability. We call this the parametersplitting method for entanglement concentration. With single-photon detectors, the parties in quantum communication can read out whether the hyper-ECPs succeed or not in principle. If none of the single-photon detectors clicks, the two hyper-ECPs succeed. The success probabilities of these two hyper-ECPs are shown in Fig. 2 and Fig. 4 respectively. It shows that the success probability of the hyper-ECP for a partially hyperentangled Bell state is determined by the two smallest parameters in the two DOFs, and the success probability of the hyper-ECP for a partially entangled two-photon cluster state is correlated to the relation of three parameters of the partially hyperentangled state.

In the second kind of condition about the two-photon four-qubit states with their parameters unknown to the two legitimate users, both the users are required to perform some quantum operations on their photons, and two copies of the unknown partially entangled states are required. In these two hyper-ECPs, the polarization parity-check measurement and the spatial-mode paritycheck measurement are performed with some PBSs and BSs, respectively. In these two hyper-ECPs, two copies of photon pairs in the unknown state are required for each round of concentration, and the two legitimate users can make their parity-check measurements synchronously. For the concentration of a partially entangled two-photon four-qubit cluster state, an auxiliary step is required to transform a nonlocal maximally hyperentangled Bell state into a nonlocal maximally entangled two-photon cluster state with a linear-optical element. These two hyper-ECPs succeed when both Alice and Bob have one single-photon detector clicked, and local phaseflip operations are needed if the outcomes of the two single-photon detectors are not in even-parity polarization modes and even-parity spatial modes. We have discussed the success probabilities of these two hyper-ECPs for some special states shown in Fig. 6. It shows that the success probabilities of the two hyper-ECPs change with the parameters of the partially hyperentangled states.

In a practical quantum communication, Alice and Bob can obtain the information about the parameters of a nonlocal partially hyperentangled pure state if they measure a sufficient number of sample photon pairs. It is not difficult to find that the two hyper-ECPs with our parameter-splitting method are more efficient and practical than those with the Schmidt projection method if there are large numbers of quantum data needed to be exchanged between the two parties in quantum communication. First, the former can be used to concentrate each of the two-photon four-qubit systems, while the latter require two copies of the two-photon four-qubit systems in the same partially entangled pure state. Second, the efficiency of the former is more than two times that of the latter. Third, the quantum circuits in the former are far simpler than those in the latter. Contrarily, if there are a small quantity of quantum data needed to be exchanged between the two parties, the hyper-ECPs with our parameter-splitting method as they do not require the two parties to measure the samples for obtaining the accurate information about the parameters of the partially hyperentangled pure state.

On one hand, although we have introduced our parameter-splitting method for the hyperentanglement concentration of two-photon four-qubit systems in nonlocal partially hyperentangled pure states with known parameters, it is obviously suitable for all the entanglement concentration on photon systems in nonlocal partially entangled pure states with known parameters, including those with one DOF and those with several DOFs, no matter what the form of the state is and what the number of the particles in the system is. The similar tasks can be accomplished with linear optics only. On the other hand, this way may be suitable for atom systems, electron-spin systems, and others if the parties in quantum communication can construct the elements similar to the UBSs and PBSs in photon systems. It is a general way for efficient entanglement concentration. If we use our parameter-splitting method for the concentration of photonic polarization entanglement, it is far simpler than the ECPs existing for photon systems in a less-entangled polarization state with known parameters, 
including those based on entanglement swapping [23], a collective unitary evolution 24], or additional single photons 25 27].

In our four hyper-ECPs, we assume the efficiency of the linear-optical elements, such as PBSs, BSs, wave plates, and half-wave plates, to be perfect. That is, there is no photon loss in these linear-optical elements. Moreover, the efficiency of the single-photon detectors is assumed to be $100 \%$. In a practical application of these hyper-ECPs, they do not work in an ideal condition. The nonideal elements and detectors will decrease the success probabilities of these hyper-ECPs. In this time, the two parties can obtain maximally hyperentangled states by postselection yet. In detail, by picking up the cases in which there is a photon emitting from each of the output ports $a_{1}\left(a_{2}\right)$ and $b_{1}\left(b_{2}\right)$, the two parties can make their hyper-ECP work in a practical condition, as the same as the entanglement purification protocols 10 12, 14, 16] and the entanglement concentration protocols [18 21] in only one DOF with linear optical elements.

In summary, we have proposed four hyper-ECPs for two classes of two-photon four-qubit states entangled in two DOFs in two kinds of conditions. With these four hyper-ECPs, the parties in quantum communication can concentrate nonlocal partially hyperentangled Bell states and nonlocal partially entangled two-photon cluster states with their parameters known or unknown to the two remote users. As hyperentangled states can increase the channel capacity of long-distance quantum communication processing, our hyper-ECPs for two-photon states entangled in two DOFs may be very useful in longdistance quantum communication in the future.

The task of entanglement concentration is to distill some quantum systems in a nonlocal maximally entangled state from those in a nonlocal partially entangled pure state for two remote users 17]. The goal of this work is focused on the nonlocal hyperentanglement concentration of two-photon four-qubit systems in a partially hyperentangled pure state. However, if the two-photon four-qubit systems are in a mixed hyperentangled state, it is more complex for the two remote users Alice and Bob to obtain some quantum systems in a hyperentangled state with a higher fidelity than the original one, as discussed in Appendix B. Of course, this is the goal of entanglement purification [8]. For a general hyperentanglement purification, nonlinearity is required [43]. As a simple example of the parameter-splitting method for the entanglement concentration of photon systems in one DOF, we discuss the concentration for polarization entangled states and spatial-mode entangled states independently in Appendix $\mathrm{C}$.

\section{ACKNOWLEDGMENTS}

This work is supported by the National Natural Science Foundation of China under Grant No. 11174039 and NECT-11-0031.
Appendix A: Hyper-ECP for two-photon four-qubit systems in an arbitrary unknown hyperentangled cluster-class state

We assume that there are four identical two-photon systems in an arbitrary partially hyperentangled cluster state,

$$
\begin{aligned}
\left|\Psi_{0}\right\rangle_{A B} & =\alpha|H\rangle_{A}|H\rangle_{B}\left|a_{1}\right\rangle\left|b_{1}\right\rangle+\beta|V\rangle_{A}|V\rangle_{B}\left|a_{1}\right\rangle\left|b_{1}\right\rangle \\
& +\gamma|H\rangle_{A}|H\rangle_{B}\left|a_{2}\right\rangle\left|b_{2}\right\rangle-\delta|V\rangle_{A}|V\rangle_{B}\left|a_{2}\right\rangle\left|b_{2}\right\rangle, \\
\left|\Psi_{0}\right\rangle_{C D} & =\alpha|H\rangle_{C}|H\rangle_{D}\left|c_{1}\right\rangle\left|d_{1}\right\rangle+\beta|V\rangle_{C}|V\rangle_{D}\left|c_{1}\right\rangle\left|d_{1}\right\rangle \\
& +\gamma|H\rangle_{C}|H\rangle_{D}\left|c_{2}\right\rangle\left|d_{2}\right\rangle-\delta|V\rangle_{C}|V\rangle_{D}\left|c_{2}\right\rangle\left|d_{2}\right\rangle, \\
\left|\Psi_{0}\right\rangle_{A^{\prime} B^{\prime}} & =\alpha|H\rangle_{A^{\prime}}|H\rangle_{B^{\prime}}\left|a_{1}^{\prime}\right\rangle\left|b_{1}^{\prime}\right\rangle+\beta|V\rangle_{A^{\prime}}|V\rangle_{B^{\prime}}\left|a_{1}^{\prime}\right\rangle\left|b_{1}^{\prime}\right\rangle \\
& +\gamma|H\rangle_{A^{\prime}}|H\rangle_{B^{\prime}}\left|a_{2}^{\prime}\right\rangle\left|b_{2}^{\prime}\right\rangle-\delta|V\rangle_{A^{\prime}}|V\rangle_{B^{\prime}}\left|a_{2}^{\prime}\right\rangle\left|b_{2}^{\prime}\right\rangle, \\
\left|\Psi_{0}\right\rangle_{C^{\prime} D^{\prime}} & =\alpha|H\rangle_{C^{\prime}}|H\rangle_{D^{\prime}}\left|c_{1}^{\prime}\right\rangle\left|d_{1}^{\prime}\right\rangle+\beta|V\rangle_{C^{\prime}}|V\rangle_{D^{\prime}}\left|c_{1}^{\prime}\right\rangle\left|d_{1}^{\prime}\right\rangle \\
& +\gamma|H\rangle_{C^{\prime}}|H\rangle_{D^{\prime}}\left|c_{2}^{\prime}\right\rangle\left|d_{2}^{\prime}\right\rangle-\delta|V\rangle_{C^{\prime}}|V\rangle_{D^{\prime}}\left|c_{2}^{\prime}\right\rangle\left|d_{2}^{\prime}\right\rangle .
\end{aligned}
$$

Here the subscripts $A B, C D, A^{\prime} B^{\prime}$, and $C^{\prime} D^{\prime}$ represent four photon pairs. The four photons $A, C, A^{\prime}$, and $C^{\prime}$ belong to Alice, and the four photons $B, D, B^{\prime}$, and $D^{\prime}$ belong to Bob. The four parameters $\alpha, \beta, \gamma$, and $\delta$ are unknown to Alice and Bob, and they satisfy the relation $|\alpha|^{2}+|\beta|^{2}+|\gamma|^{2}+|\delta|^{2}=1$.

The principle of our hyper-ECP for an arbitrary partially hyperentangled two-photon four-qubit cluster state with unknown parameters includes three steps. Let us describe them in detail below.

First, Alice and Bob divide the four photon pairs into two groups, that is, $A B$ and $C D$ in one group, and $A^{\prime} B^{\prime}$ and $C^{\prime} D^{\prime}$ in the other group. Alice and Bob perform the same operations on these two groups. The quantum circuit shown in Fig. 5 without all the half-wave plates $R_{90}$ is used to select the terms with the same parameter, resorting to its parity-check effect. The even-parity terms of the polarization DOF of photon pairs in Alice's hand and the odd-parity terms of the spatial-mode DOF of photon pairs in Bob's hand are picked up. After the detections on $C D\left(C^{\prime} D^{\prime}\right)$ and the local phase-flip operations on the photon $B\left(B^{\prime}\right)$, the states of the photon pairs $A B$ and $A^{\prime} B^{\prime}$ become

$$
\begin{aligned}
\left|\Psi_{1}\right\rangle_{A B}= & \frac{|\alpha \gamma|}{\sqrt{2\left(|\alpha \gamma|^{2}+|\beta \delta|^{2}\right)}}\left(\left|a_{1}\right\rangle\left|b_{1}\right\rangle+\left|a_{2}\right\rangle\left|b_{2}\right\rangle\right) \\
& \otimes\left(|H\rangle_{A}|H\rangle_{B}-\frac{\beta \delta}{\alpha \gamma}|V\rangle_{A}|V\rangle_{B}\right) \\
\left|\Psi_{1}\right\rangle_{A^{\prime} B^{\prime}}= & \frac{|\alpha \gamma|}{\sqrt{2\left(|\alpha \gamma|^{2}+|\beta \delta|^{2}\right)}}\left(\left|a_{1}^{\prime}\right\rangle\left|b_{1}^{\prime}\right\rangle+\left|a_{2}^{\prime}\right\rangle\left|b_{2}^{\prime}\right\rangle\right) \\
& \otimes\left(|H\rangle_{A^{\prime}}|H\rangle_{B^{\prime}}-\frac{\beta \delta}{\alpha \gamma}|V\rangle_{A^{\prime}}|V\rangle_{B^{\prime}}\right) .
\end{aligned}
$$

Now, the spatial modes of the two-photon systems are in a maximally entangled state, and the next step is to let the polarization modes become a maximally entangled one. This task can be completed with the quantum circuit shown in Fig. 5. The polarization parity-check 
measurement is performed on the photon pair $A A^{\prime}$ in Alice's hand, and the spatial-mode parity-check measurement is performed on the photon pair $B B^{\prime}$ in Bob's hand. Then the even-parity terms of the polarization DOF of photon pairs in Alice's hand and the odd-parity terms of the spatial-mode DOF of photon pairs in Bob's hand are picked up. After the detections on $A^{\prime} B^{\prime}$ and the local phase-flip operations on the photon $B$, the state of the photon pair $A B$ becomes $\left|\varphi_{f}\right\rangle_{A B}=\frac{1}{2}\left(|H\rangle_{A}|H\rangle_{B}+\right.$ $\left.|V\rangle_{A}|V\rangle_{B}\right)\left(\left|a_{1}\right\rangle\left|b_{1}\right\rangle+\left|a_{2}\right\rangle\left|b_{2}\right\rangle\right)$ which is a maximally hyperentangled Bell state. This maximally hyperentangled Bell state can be transformed into a maximally entangled two-photon cluster state $\left|\psi_{f}\right\rangle_{A B}=\frac{1}{2}\left(|H\rangle_{A}|H\rangle_{B}\left|a_{1}\right\rangle\left|b_{1}\right\rangle+\right.$ $\left.|V\rangle_{A}|V\rangle_{B}\left|a_{1}\right\rangle\left|b_{1}\right\rangle+|H\rangle_{A}|H\rangle_{B}\left|a_{2}\right\rangle\left|b_{2}\right\rangle-|V\rangle_{A}|V\rangle_{B}\left|a_{2}\right\rangle\left|b_{2}\right\rangle\right)$ with the operation shown in Fig. 7.

Up to now, we have transformed an arbitrary partially entangled two-photon cluster state into a maximally hyperentangled two-photon cluster state with the success probability $P_{4}=\frac{4|\alpha \beta \gamma \delta|^{2}}{2\left(|\alpha \gamma|^{2}+|\delta \beta|^{2}\right)}$ by exploiting some linear-optical elements only. The principle of the first two steps of our hyper-ECP for partially hyperentangled two-photon cluster states is the same as that in the hyper-ECP for partially hyperentangled Bell states, and an arbitrary partially entangled two-photon cluster state can be changed to be a maximally hyperentangled Bell one. In the third step, the maximally hyperentangled Bell state is transformed into a maximally entangled twophoton cluster state with a linear-optical element.

In this hyper-ECP, one can see that four photon pairs are required for concentrating a hyperentangled twophoton four-qubit cluster-class state with four arbitrary unknown parameters. On one hand, it is not efficient, compared with the hyper-ECP for a partially hyperentangled Bell state as the latter requires only two pairs in a practical application. Moreover, this is, in principle, the necessary condition for the hyperentanglement concentration of an arbitrary unknown two-photon fourqubit cluster state because there are three independent parameters which are unknown to Alice and Bob, while there are only two independent parameters which are unknown to Alice and Bob in the hyperentanglement concentration of an unknown partially hyperentangled Bell state. On the other hand, it is possible to prepare a nonlocal maximally hyperentangled cluster-class state with a nonlocal maximally hyperentangled Bell state using a linear-optical element shown in Fig. 7. Therefore, in a practical application in quantum communication, the hyper-ECP for the hyperentangled Bell state is sufficient for long-distance quantum communication with hyperentangled two-photon four-qubit states. After the parties obtain a nonlocal maximally hyperentangled Bell state, they need only transform it into a nonlocal maximally hyperentangled cluster state with linear-optical elements.
Appendix B: Entanglement purification for a spatial-polarization mixed hyperentangled Bell state

We assume that there are two identical two-photon four-qubit systems in a mixed hyperentangled state,

$$
\begin{aligned}
\rho_{A B}= & {\left[F_{1}\left|\phi_{A B}^{+}\right\rangle_{P}\left\langle\phi_{A B}^{+}\left|+\left(1-F_{1}\right)\right| \psi_{A B}^{+}\right\rangle_{P}\left\langle\psi_{A B}^{+}\right|\right] } \\
& \otimes\left|\phi_{A B}^{+}\right\rangle_{S}\left\langle\phi_{A B}^{+}\right|, \\
\rho_{C D}= & {\left[F_{1}\left|\phi_{C D}^{+}\right\rangle_{P}\left\langle\phi_{C D}^{+}\left|+\left(1-F_{1}\right)\right| \psi_{C D}^{+}\right\rangle_{P}\left\langle\psi_{C D}^{+}\right|\right] } \\
& \otimes\left|\phi_{C D}^{+}\right\rangle_{S}\left\langle\phi_{C D}^{+}\right| .
\end{aligned}
$$

Here the subscripts $A B$ and $C D$ represent two photon pairs. The two photons $A$ and $C$ belong to Alice, and the two photons $B$ and $D$ belong to Bob. The subscripts $P$ and $S$ represent the polarization and the spatial-mode DOFs, respectively. The four states $\left|\phi^{+}\right\rangle_{P},\left|\psi^{+}\right\rangle_{P},\left|\phi^{+}\right\rangle_{S}$, and $\left|\psi^{+}\right\rangle_{S}$ are defined as

$$
\begin{aligned}
\left|\phi^{+}\right\rangle_{P} & =\frac{1}{\sqrt{2}}(|H H\rangle+|V V\rangle), \\
\left|\psi^{+}\right\rangle_{P} & =\frac{1}{\sqrt{2}}(|H V\rangle+|V H\rangle), \\
\left|\phi^{+}\right\rangle_{S} & =\frac{1}{\sqrt{2}}\left(\left|a_{1} b_{1}\right\rangle+\left|a_{2} b_{2}\right\rangle\right), \\
\left|\psi^{+}\right\rangle_{S} & =\frac{1}{\sqrt{2}}\left(\left|a_{1} b_{2}\right\rangle+\left|a_{2} b_{1}\right\rangle\right) .
\end{aligned}
$$

The state of the four-photon system $A B C D$ can be described as

$$
\rho=\rho_{A B} \otimes \rho_{C D}
$$

This mixed hyperentangled Bell state can be viewed as the mixture of four maximally hyperentangled Bell states, the same as the conventional entanglement purification protocols for photon pairs $[8-16]$. That is, it is the mixture of the four pure states in the polarization DOF: $\left|\phi_{A B}^{+}\right\rangle_{P} \otimes\left|\phi_{C D}^{+}\right\rangle_{P}$ with the probability $F_{1}^{2},\left|\phi_{A B}^{+}\right\rangle_{P} \otimes$ $\left|\psi_{C D}^{+}\right\rangle_{P}$ with the probability $F_{1}\left(1-F_{1}\right),\left|\psi_{A B}^{+}\right\rangle_{P} \otimes\left|\phi_{C D}^{+}\right\rangle_{P}$ with the probability $F_{1}\left(1-F_{1}\right)$, and $\left|\psi_{A B}^{+}\right\rangle_{P} \otimes\left|\psi_{C D}^{+}\right\rangle_{P}$ with the probability $\left(1-F_{1}\right)^{2}$. The state of the system in the spatial-mode DOF is $\left|\phi_{A B}^{+}\right\rangle_{S} \otimes\left|\phi_{C D}^{+}\right\rangle_{S}$.

In order to implement this hyper-EPP, Alice and Bob have to perform the same operations on the two photon pairs $A C$ and $B D$, shown in Fig. 8. That is, Alice (Bob) performs the polarization parity-check measurements and the spatial-mode parity-check measurements on the photon pair $A C(B D)$ first and then detects the photon $C(D)$. If both Alice and Bob detect one and only one photon with their single-photon detectors, the state of the two-photon system $A B$ becomes

$$
\begin{aligned}
\rho_{f}= & {\left[F_{1}^{\prime}\left|\phi^{+}\right\rangle_{P}\left\langle\phi^{+}\left|+\left(1-F_{1}^{\prime}\right)\right| \psi^{+}\right\rangle_{P}\left\langle\psi^{+}\right|\right]_{A B} } \\
& \otimes\left|\phi^{+}\right\rangle_{S}\left\langle\left.\phi^{+}\right|_{A B} .\right.
\end{aligned}
$$

Here $F_{1}^{\prime}=F_{1}^{2} /\left[F_{1}^{2}+\left(1-F_{1}\right)^{2}\right]$. If $F_{1}>1 / 2, F_{1}^{\prime}>F_{1}$. That is, the fidelity of the maximally hyperentangled 


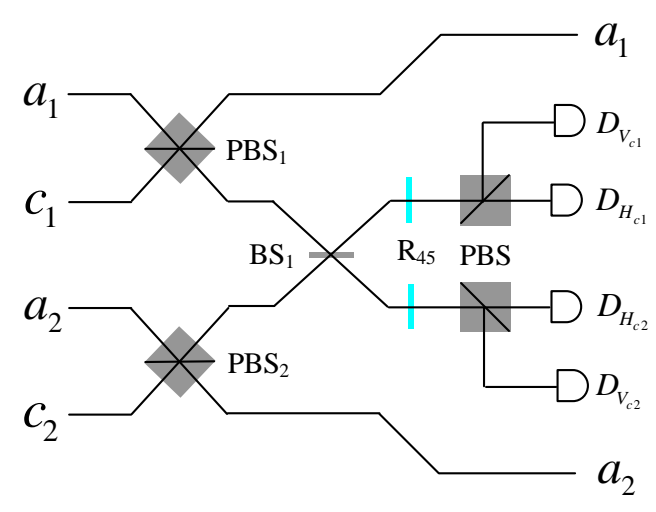

FIG. 8: (Color online) Schematic diagram of our hyperentanglement purification protocol (hyper-EPP) for a mixed hyperentangled Bell state with polarization bit-flip errors. Bob performs the same operations as Alice by replacing the photons $A$ and $C$ with the photons $B$ and $D$, respectively.

Bell state $\left|\varphi_{f}\right\rangle_{A B}=\frac{1}{2}\left(|H\rangle_{A}|H\rangle_{B}+|V\rangle_{A}|V\rangle_{B}\right)\left(\left|a_{1}\right\rangle\left|b_{1}\right\rangle+\right.$ $\left.\left|a_{2}\right\rangle\left|b_{2}\right\rangle\right)$ increases after a round of entanglement purification if $F_{1}>1 / 2$. In a practical application, Alice and Bob should resort to postselection for judging whether there is one and only one photon detected by each of them or not, similar to the conventional EPPs in the polarization DOF [8-13].

Our hyper-EPP is used to purify the mixed hyperentangled Bell state with polarization bit-flip errors. The polarization phase-flip error can be transformed into the polarization bit-flip error with local operations [8-13]. The spatial-mode entanglement is robust against bit-flip errors because it is hard for a photon to permeate into another fiber [11, 12]. The phase-flip error of the spatialmode DOF can be depressed with current technology [11, 12]. If there are both the polarization bit-flip error and the spatial-mode bit-flip error in a mixed hyperentangled Bell state, the present hyper-EPP cannot be implemented with linear optics, which is limited by the ability of linear optics for purifying only one qubit error. In this time, we should use nonlinear optics to implement the hyper-EPP for a mixed hyperentangled Bell state with both the polarization bit-flip error and the spatial-mode bit-flip error [43].

Appendix C: Parameter-splitting-based entanglement concentration of photon systems in a known partially entangled state in one DOF

In this section, we will describe the ECPs for a partially entangled polarization Bell state and a partially entangled spatial-mode Bell state independently based on our parameter-splitting method in detail.

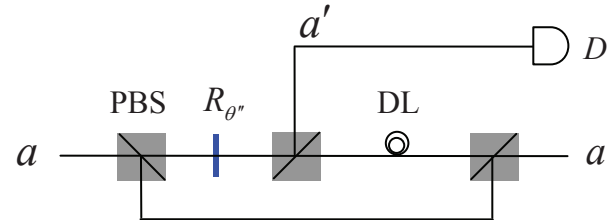

FIG. 9: (Color online) Schematic diagram of our parametersplitting-based ECP for a partially entangled Bell state with known parameters in the polarization DOF. $R_{\theta^{\prime \prime}}$ represents a wave plate which can rotate the horizontal polarization with an angle $\theta^{\prime \prime}=\arccos (\beta / \alpha)$. $D$ represents a single-photon detector.

\section{Parameter-splitting-based entanglement concentration of photon systems in a known partially entangled polarization state}

Let us assume that the initial partially entangled Bellclass state in the polarization DOF is

$$
\left|\varphi_{0}\right\rangle_{A B}=\alpha|H\rangle_{A}|H\rangle_{B}+\beta|V\rangle_{A}|V\rangle_{B} .
$$

Here the subscripts $A$ and $B$ represent the two photons shared by the two remoter users Alice and Bob, respectively. $\alpha$ and $\beta$ are two real parameters that are known to Alice and Bob, and they satisfy the relation $|\alpha|^{2}+|\beta|^{2}=1$ and $|\alpha|>|\beta|$.

The principle of the ECP for a partially entangled polarization Bell state is shown in Fig. 9, which can be implemented by performing local unitary operations on the photon $A$ in its polarization DOF. Alice performs the polarization unitary operation $R_{\theta^{\prime \prime}}$ on the photon $A$, which rotates the horizontal polarization $|H\rangle$ with an angle $\theta^{\prime \prime}=\arccos (\beta / \alpha)$, that is, $|H\rangle \rightarrow \cos \theta^{\prime \prime}|H\rangle+\sin \theta^{\prime \prime}|V\rangle$. After the photon $A$ passes through PBS and $R_{\theta^{\prime \prime}}$, the state of the system is transformed from $\left|\varphi_{0}\right\rangle_{A B}$ into $\left|\varphi_{1}\right\rangle_{A B}$. Here

$$
\begin{aligned}
\left|\varphi_{1}\right\rangle_{A B}= & \frac{1}{\sqrt{2}}\left[\beta\left(|H\rangle_{A}|H\rangle_{B}+|V\rangle_{A}|V\rangle_{B}\right)\right. \\
& \left.+\sqrt{|\alpha|^{2}-|\beta|^{2}}\left|V^{\prime}\right\rangle_{A}|H\rangle_{B}\right] .
\end{aligned}
$$

Here $\left|V^{\prime}\right\rangle$ presents the vertical polarization of the photon after the operation $R_{\theta^{\prime \prime}}$, and the photon will emit from the spatial mode $a^{\prime}$ and it will be detected by the singlephoton detector $D$ in principle. If the detector $D$ does not click, the state $\left|\varphi_{1}\right\rangle_{A B}$ becomes a maximally entangled one. That is, the state of the two-photon system $A B$ becomes

$$
\left|\phi^{+}\right\rangle_{A B}=\frac{1}{\sqrt{2}}(|H\rangle|H\rangle+|V\rangle|V\rangle)_{A B} .
$$

This is just the maximally entangled polarization Bell state of a two-photon system. If the detector $D$ clicks, the polarization state $\left|\varphi_{1}\right\rangle_{A B}$ is projected into the product state $\left|V^{\prime}\right\rangle_{A}|H\rangle_{B}$. Certainly, the photon $A$ is destroyed, and the entanglement concentration fails in this time. 
Now we have implemented our parameter-splittingbased ECP for a partially entangled polarization Bell state with known parameters. If the detector $D$ does not click, our ECP succeeds, which takes place with the probability of $P_{p}=2|\beta|^{2}$. If the detector $D$ clicks, the photon $A$ is destroyed and our ECP fails.

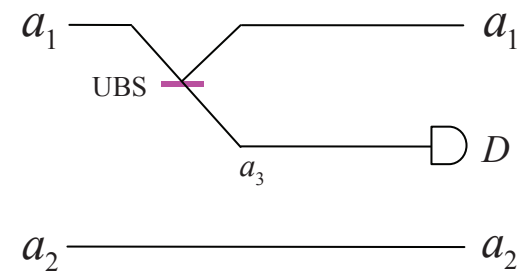

FIG. 10: (Color online) Schematic diagram of our parametersplitting-based ECP for a partially entangled Bell state with known parameters in the spatial-mode DOF. UBS represents an unbalanced beam splitter with the reflection coefficient $R=\beta / \alpha$.

\section{Parameter-splitting-based entanglement concentration of photon systems in a known partially entangled spatial-mode state}

Let us assume that the initial partially entangled Bellclass state in the spatial-mode DOF is

$$
\left|\varphi_{0}\right\rangle_{A B}=\alpha\left|a_{1}\right\rangle\left|b_{1}\right\rangle+\beta\left|a_{2}\right\rangle\left|b_{2}\right\rangle \text {. }
$$

Here the subscripts $A$ and $B$ represent the two photons shared by Alice and Bob. $\alpha$ and $\beta$ are two real parameters that are known to Alice and Bob, and they satisfy the relation $|\alpha|^{2}+|\beta|^{2}=1$ and $|\alpha|>|\beta|$.

The principle of the ECP for a partially entangled spatial-mode Bell state is shown in Fig. 10. It can be implemented by performing local unitary operations on the photon $A$ in the spatial-mode DOF. Alice performs a unitary operation on the spatial mode $a_{1}$ by using an unbalanced BS [42] with the reflection coefficient $R=\beta / \alpha$, shown in Fig. 1(b), and the partially entangled spatialmode Bell state $\left|\varphi_{0}\right\rangle_{A B}$ is changed to be $\left|\varphi_{1}\right\rangle_{A B}$. Here

$$
\begin{aligned}
\left|\varphi_{1}\right\rangle_{A B}= & \beta\left(\left|a_{1}\right\rangle\left|b_{1}\right\rangle+\left|a_{2}\right\rangle\left|b_{2}\right\rangle\right) \\
& +\sqrt{|\alpha|^{2}-|\beta|^{2}}\left|a_{3}\right\rangle\left|b_{1}\right\rangle .
\end{aligned}
$$

The state of the two-photon system $A B$ becomes a maximally entangled one if the detector $D$ does not click. That is, the state of the two-photon system $A B$ becomes

$$
\left|\varphi_{2}\right\rangle_{A B}=\frac{1}{\sqrt{2}}\left(\left|a_{1}\right\rangle\left|b_{1}\right\rangle+\left|a_{2}\right\rangle\left|b_{2}\right\rangle\right) .
$$

If the detector $D$ clicks, the state of the system is projected into a product state $\left|a_{3}\right\rangle\left|b_{2}\right\rangle$. In this time, the photon $A$ is destroyed, and the entanglement concentration fails.

It is not difficult to calculate the success probability of our ECP for a partially entangled spatial-mode Bell state. If the detector $D$ does not click, our ECP succeeds, which takes place with the probability of $P_{s}=2|\beta|^{2}$. If the detector $D$ clicks, the photon $A$ is destroyed and our ECP fails.
[1] M. A. Nielsen and I. L. Chuang, Quantum Computation and Quantum Information, Cambridge University Press, Cambridge, (2000).

[2] N. Gisin, G. Ribordy, W. Tittel, and H. Zbinden, Rev. Mod. Phys. 74, 145 (2002).

[3] C. H. Bennett, G. Brassard, C. Crépeau, R. Jozsa, A. Peres, and W. K. Wootters, Phys. Rev. Lett. 70 , 1895 (1993).

[4] C. H. Bennett and S. J. Wiesner, Phys. Rev. Lett. 69, 2881 (1992).

[5] X. S. Liu, G. L. Long, D. M. Tong, and F. Li, Phys. Rev. A 65, 022304 (2002).

[6] M. Hillery, V. Bužek, and A. Berthiaume, Phys. Rev. A 59, 1829 (1999).

[7] L. Xiao, G. L. Long, F. G. Deng, and J. W. Pan, Phys. Rev. A 69, 052307 (2004).

[8] C. H. Bennett, G. Brassard, S. Popescu, B. Schumacher, J. A. Smolin, and W. K. Wootters, Phys. Rev. Lett. 76, 722 (1996).

[9] D. Deutsch, A. Ekert, R. Jozsa, C. Macchiavello, S. Popescu, and A. Sanpera, Phys. Rev. Lett. 77, 2818 (1996).
[10] J. W. Pan, C. Simon, Č. Brukner and A. Zeilinger, Nature (London) 410, 1067 (2001).

[11] C. Simon and J. W. Pan, Phys. Rev. Lett. 89, 257901 (2002).

[12] J. W. Pan, S. Gasparonl, R. Ursin, G. Weihs, and A. Zellinger, Nature (London) 423, 417 (2003).

[13] Y. B. Sheng, F. G. Deng, and H. Y. Zhou, Phys. Rev. A 77, 042308 (2008).

[14] Y. B. Sheng and F. G. Deng, Phys. Rev. A 81, 032307 (2010).

[15] Y. B. Sheng and F. G. Deng, Phys. Rev. A 82, 044305 (2010).

[16] F. G. Deng, Phys. Rev. A 83, 062316 (2011).

[17] C. H. Bennett, H. J. Bernstein, S. Popescu, and B. Schumacher, Phys. Rev. A 53, 2046 (1996).

[18] T. Yamamoto, M. Koashi, and N. Imoto, Phys. Rev. A 64, 012304 (2001).

[19] Z. Zhao, J. W. Pan, and M. S. Zhan, Phys. Rev. A 64, 014301 (2001).

[20] Z. Zhao, T. Yang, Y. A. Chen, A. N. Zhang, and J. W. Pan, Phys. Rev. Lett. 90, 207901 (2003).

[21] T. Yamamoto, M. Koashi, S K. Ozdemir, and N. Imoto, 
Nature (London) 421, 343 (2003).

[22] Y. B. Sheng, F. G. Deng, and H. Y. Zhou, Phys. Rev. A 77, 062325 (2008).

[23] S. Bose, V. Vedral, and P. L. Knight, Phys. Rev. A 60, 194 (1999).

[24] B. S. Shi, Y. K. Jiang, and G. C. Guo, Phys. Rev. A 62, 054301 (2000).

[25] Y. B. Sheng, L. Zhou, S. M. Zhao, and B. Y. Zheng, Phys. Rev. A 85, 012307 (2012).

[26] F. G. Deng, Phys. Rev. A 85, 022311 (2012).

[27] Y. B. Sheng, L. Zhou, and S. M. Zhao, Phys. Rev. A 85, 042302 (2012).

[28] P. G. Kwiat and H. Weinfurter, Phys. Rev. A 58, R2623 (1998).

[29] S. P. Walborn, S. Pádua, and C. H. Monken, Phys. Rev. A 68, 042313 (2003).

[30] C. Schuck, G. Huber, C. Kurtsiefer, and H. Weinfurter, Phys. Rev. Lett. 96, 190501 (2006).

[31] M. Barbieri, G. Vallone, P. Mataloni, and F. De Martini, Phys. Rev. A 75, 042317 (2007).

[32] S. P. Walborn, W. A. T. Nogueira, S. Ṕadua, and C. H. Monken, Europhys. Lett. 62, 161 (2003).
[33] J. T. Barreiro, N. K. Langford, N. A. Peters, and P. G. Kwiat, Phys. Rev. Lett. 95, 260501 (2005).

[34] J. T. Barreiro, T. C. Wei, and P. G. Kwiat, Nature Phys. 4, 282 (2008).

[35] Y. B. Sheng, F. G. Deng, and G. L. Long, Phys. Rev. A 82, 032318 (2010).

[36] B. C. Ren, H. R. Wei, M. Hua, T. Li, and F. G. Deng, Opt. Express 20, 24664 (2012).

[37] T. J. Wang, Y. Lu, and G. L. Long, Phys. Rev. A 86, 042337 (2012).

[38] T. C. Wei, J. T. Barreiro, and P. G. Kwiat, Phys. Rev. A 75, 060305 (2007).

[39] T. J. Wang, S. Y. Song, and G. L. Long, Phys. Rev. A 85, 062311 (2012).

[40] T. M. Graham, J. T. Barreiro, M. Mohseni, and P. G. Kwiat, Phys. Rev. Lett. 110, 060404 (2013).

[41] C. K. Hong, Z. Y. Ou, and L. Mandel, Phys. Rev. Lett. 59, 2044 (1987).

[42] M. Reck, A. Zeilinger, H. J. Bernstein, and P. Bertani, Phys. Rev. Lett. 73, 58 (1994).

[43] B. C. Ren and F. G. Deng, unpublished. 